\title{
Epilepsia
}

\section{Strong coupling between slow oscillations and wide fast ripples in children with epileptic spasms: Investigation of modulation index and occurrence rate}

\author{
Yasushi Iimura $^{1}$ (D) | Kevin Jones ${ }^{2}$ | Lynne Takada ${ }^{1}$ | Itsuki Shimizu ${ }^{1}$ | Misaki Koyama ${ }^{1}$ | \\ Kyoko Hattori $^{1}$ | Yushi Okazawa ${ }^{1}$ | Yutaka Nonoda ${ }^{3}$ | Eishi Asano ${ }^{3}$ (D) | \\ Tomoyuki Akiyama $^{4}$ | Cristina Go ${ }^{1}$ | Ayako Ochi ${ }^{1}$ | O. Carter Snead III ${ }^{1}$ | \\ Elizabeth J. Donner $^{1}$ | James T. Rutka ${ }^{5}$ | James M. Drake ${ }^{5}$ Hiroshi Otsubo ${ }^{1}$
}

${ }^{1}$ Division of Neurology, Hospital for Sick Children, Toronto, Ontario, Canada

${ }^{2}$ Division of Neurology, Department of Pediatrics, McMaster Children's Hospital, Hamilton, Ontario, Canada

${ }^{3}$ Pediatrics and Neurology, Children's Hospital of Michigan, Wayne State University, Detroit, MI, USA

${ }^{4}$ Department of Child Neurology, Okayama University Hospital, Okayama, Japan

${ }^{5}$ Division of Neurosurgery, Hospital for Sick Children, Toronto, Ontario, Canada

\section{Correspondence}

Yasushi Iimura, Division of Neurology, Hospital for Sick Children, Toronto, Ontario, Canada.

Email: yasuyasu0104@gmail.com

Funding information EpiLink - the Epilepsy Research Program of the Ontario Brain Institute

\section{Summary}

Objective: Epileptic spasms (ES) often become drug-resistant. To reveal the electrophysiological difference between children with ES (ES+) and without ES (ES-), we compared the occurrence rate (OR) of high-frequency oscillations (HFOs) and the modulation index (MI) of coupling between slow and fast oscillations. In ES+, we hypothesized that (1) pathological HFOs are more widely distributed and (2) slow oscillations show stronger coupling with pathological HFOs than in ES-.

Methods: We retrospectively reviewed 24 children with drug-resistant multilobar onset epilepsy, who underwent intracranial video electroencephalography prior to multilobar resections. We measured the OR of HFOs and determined the electrodes with a high rate of HFOs by cluster analysis. We calculated MI, which reflects the degree of coupling between HFO (ripple/fast ripple [FR]) amplitude and 5 different frequency bands of delta and theta activities $(0.5-1 \mathrm{~Hz}, 1-2 \mathrm{~Hz}, 2-$ $3 \mathrm{~Hz}, 3-4 \mathrm{~Hz}, 4-8 \mathrm{~Hz})$.

Results: In ES+ $(\mathrm{n}=10)$, the $\mathrm{OR}_{(\mathrm{FRs})}$, the number of electrodes with high-rate FRs, and the $\mathrm{MI}_{(\mathrm{FRs}}$ \& 3-4 $\left.\mathrm{Hz}\right)$ in all electrodes were significantly higher than in ES - $(n=14)$. In both the ES+ and ES- groups, $\mathrm{MI}_{\text {(ripples/FRs \& 3-4 Hz) }}$ was the highest among the 5 frequency bands. Within the good seizure outcome group, the $\mathrm{OR}_{(\mathrm{FRs})}$ and the $\left.\mathrm{MI}_{(\mathrm{FRs}} \& 3-4 \mathrm{~Hz}\right)$ in the resected area in ES+ were significantly higher than in $\left.\mathrm{ES}-\left(\mathrm{OR}_{[\mathrm{FRs}]}, P=.04 ; \mathrm{MI}_{[\mathrm{FRs}} \& 3-4 \mathrm{~Hz}\right], P=.04\right)$.

Significance: In ES+, the larger number of high-rate FR electrodes indicates more widespread epileptogenicity than in $\mathrm{ES}-$. High values of $\mathrm{OR}_{(\mathrm{FRs})}$ and $\left.\mathrm{MI}_{(\mathrm{FRs}} \& 3-4 \mathrm{~Hz}\right)$ in ES+ compared to ES - are a signature of the severity of epileptogenicity. We proved that ES+ children who achieved seizure freedom following multilobar resections exhibited strong coupling between slow oscillations and FRs.

\section{K E Y W O R D S}

drug-resistant epilepsy, epilepsy surgery, focal seizure, intracranial EEG, multilobar resection 


\section{1 | INTRODUCTION}

\section{1 | Epileptic spasms}

Epileptic spasms (ES) are characterized by a brief and sudden flexion, extension, or mixed movement, either symmetric or asymmetric, lasting for 1-2 seconds. ${ }^{1}$ ES are the major subgroup of seizure types in infants from 0 to 2 years old. ${ }^{2}$ The underlying mechanism for the generation of ES is not yet known. International League Against Epilepsy (ILAE) 2017 classification of seizure types categorized ES into focal, generalized, or combined onset seizure type. ${ }^{3}$ The cessation of drug-resistant ES after multilobar resection including subtotal hemispherectomy indicates that one of the mechanisms of ES is the focal onset seizure of cortical origin. ${ }^{4-7}$ Barba et al reported 58 patients with ES who underwent unilobar resection and 22 patients with ES who underwent multilobar resection. Forty-nine of the 80 patients became seizure-free. ${ }^{8}$

There have been no reports comparing the electrophysiological findings between ES (ES+ group) and focal seizures without ES (ES- group).

\section{2 | High-frequency oscillations}

High-frequency oscillations (HFOs) are classified into ripples at $80-200 \mathrm{~Hz}$ and fast ripples (FRs) at $>200 / 250 \mathrm{~Hz} .{ }^{9}$ HFOs, especially FRs, are estimated to be a potential marker for epileptogenicity. ${ }^{10-13}$ A meta-analysis of 11 papers by Höller et al provides evidence of a higher resection ratio of ripples (10 studies) and/or FRs (7 studies) in seizure-free patients compared with non-seizure-free patients. ${ }^{13}$ Kobayashi et al reported ictal fast oscillations $(40-150 \mathrm{~Hz})$ on the scalp video electroencephalogram (SVEEG) in infantile spasm patients to demonstrate the cortical origin of ES. ${ }^{14,15}$ There have been no reports examining HFOs on intracranial electroencephalogram (EEG) comparing ES+ and ES- .

\section{3 | Modulation index}

Modulation index (MI) is one of the parameters that represent the strength of phase-amplitude coupling between the HFO amplitude and the slow oscillations. ${ }^{16}$ Pathological HFOs may be preferentially coupled with slow oscillations at $3-4 \mathrm{~Hz}$ more than with slow oscillations at $0.5-1 \mathrm{~Hz}$, which are more often coupled with physiological HFOs. ${ }^{17}$ $\mathrm{MI}_{\text {(ripples/FRs \& 3-4 Hz) }}$ has been shown to differentiate the multilobar epileptogenic zones from the motor area. ${ }^{7}$

\section{4 | Hypothesis}

We tested the hypotheses that in ES+ children, as compared to ES- children, (1) pathological HFOs are more

\section{Key Points}

- Children with epileptic spasms (ES+) present a significantly larger number of electrodes with high-rate fast ripples

- $\mathrm{MI}_{\text {(ripples/FRs \& } 5 \text { frequency bands) }}$ was higher in ES+ than in ES-

- ES+ and ES- children are distinguished by higher values of $\mathrm{OR}_{(\mathrm{FRs})}$ and $\mathrm{MI}_{(\mathrm{FRs}}$ \& 3-4 $\left.\mathrm{Hz}\right)$ in the resected area that correspond with the severity of epileptogenicity

- Strong coupling of slow oscillations and FRs in ES+ suggests that multilobar resection may be required in a subset of ES children

widely distributed, and (2) slow oscillations show stronger coupling with pathological HFOs. The objective of our study is to elucidate the extent and the severity of the epileptogenicity in the ES+ group versus the ES- group.

\section{MATERIALS AND METHODS}

\section{1 | Patients}

We retrospectively reviewed 24 children with drug-resistant multilobar onset epilepsy who underwent intracranial video EEG (IVEEG) recordings for presurgical evaluation between June 2009 and December 2013 at the Hospital for Sick Children. Before surgery, all 24 children underwent SVEEG, magnetic resonance imaging (MRI), and magnetoencephalography (MEG). We assessed 10 ES+ children and 14 ES - children. This study was approved by the Research Ethics Board of the Hospital for Sick Children.

\section{2 | IVEEG recording}

We selected the region to implant the subdural grid electrodes based on the seizure semiology, ictal/interictal data on SVEEG, MRI lesions, and MEG spike dipoles. We performed IVEEG with subdural grid electrodes and several strip and/or depth electrodes. We implanted the intracranial electrodes in the same way as reported previously. ${ }^{12,18} \mathrm{We}$ used subdural grid electrodes (diameter $=4 \mathrm{~mm}$, exposure $=2.3 \mathrm{~mm}$, effective surface area $=4.2 \mathrm{~mm}^{2}$, spacing $=9$ $13 \mathrm{~mm}$ ) and depth electrodes (effective surface area $=$ $8.3 \mathrm{~mm}^{2}$, spacing $=7 \mathrm{~mm}$; Ad-Tech Medical Instrument Corporation, Racine, WI, USA). We recorded IVEEG by using HARMONY 5.4 (Stellate, Montreal, Quebec, Canada) at a sampling rate of $1 \mathrm{kHz}(\mathrm{n}=6)$ or $2 \mathrm{kHz}$ $(\mathrm{n}=18)$. Our IVEEG data were acquired using 72-121 
(mean \pm standard deviation $[\mathrm{SD}]=106 \pm 13$ ) electrodes for 46-98 hours (mean $=78$ hours).

\subsection{Determination of resection margin}

We determined the resection margin based on the visual and spectral analyses of interictal epileptiform discharges on IVEEG, ictal findings on IVEEG, equivalent current dipoles for the interictal epileptiform discharges on MEG, and the location of eloquent areas. ${ }^{18}$ The data in this study were retrospectively analyzed; thus, the results of this study did not affect the real-time surgical decision making.

\section{4 | EEG selection for analysis}

We selected 5 epochs of 5-minute interictal IVEEG during slow-wave sleep. ${ }^{19}$ The 5 epochs were remote from each other and from seizures by at least 1 hour. Interictal HFOs can be observed most frequently during non-rapid eye movement sleep. ${ }^{20,21}$

\section{5 | Seizure outcome}

Postsurgical seizure outcome was evaluated according to ILAE classification. ${ }^{22}$ We defined the children with class I to II as the "good seizure outcome group" and the children with class III to VI as the "poor seizure outcome group."

\section{6 | Occurrence rate of HFOs}

We detected interictal ripples $(80-200 \mathrm{~Hz})$ and FRs $(>200 \mathrm{~Hz}$ ) during slow-wave sleep using bipolar montage with pairs of 2 adjacent electrodes in successive numbers connected, excluding the reference electrodes and those with significant artifact. $^{23}$ We visually inspected each EEG epoch to ensure that they were not contaminated by significant artifacts, such as environmental or muscle artifacts. We acquired the occurrence rate $(\mathrm{OR})$ of ripples $\left(\mathrm{OR}_{\text {[ripples] }}\right)$ and the $\mathrm{OR}$ of FRs $\left(\mathrm{OR}_{[\mathrm{FRs}]}\right)$ using the automated detection of HFOs by MATLAB (R2011b; MathWorks, Natick, MA, USA; Figure S1). ${ }^{12}$ We generated topographic maps demonstrating the distribution of $\mathrm{OR}_{\text {(ripples/FRs) }}$ (Figure 1).

We acquired 5 values of $\mathrm{OR}_{\text {(ripples/FRs) }}$ for each electrode by the analysis of 5 interictal EEG epochs. We calculated the mean of the $5 \mathrm{OR}_{\text {(ripples/FRs) }}$ per each electrode to demonstrate the $\mathrm{OR}_{\text {(ripples/FRs). }}$.

We categorized the electrodes into 3 clusters: high-rate ripples/FRs, medium-rate ripples/FRs, and low-rate ripples/ FRs, according to the $\mathrm{OR}_{\text {(ripples/FRs) }}$ by Ward's method, which is a frequently used hierarchical clustering method,

\section{Topographic maps of occurrence rate (OR: / $\mathrm{min}$ ) of high frequency oscillations and the resection area}
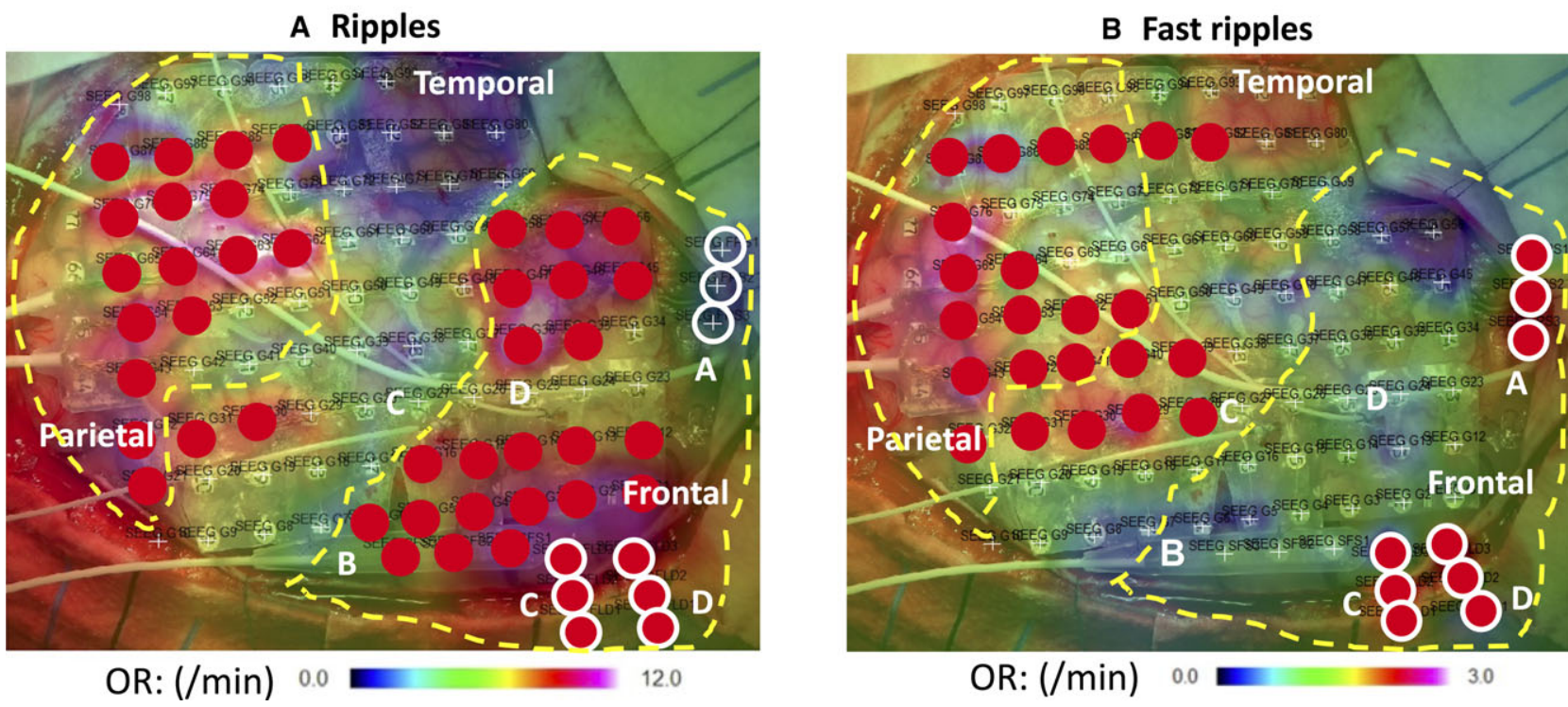

FIG URE 1 Topographic maps of occurrence rate (OR; per minute) of high-frequency oscillations and the resection area. The topographic maps show the ORs of ripples (A), and fast ripples (FRs; B) in Case \#7 in the epileptic spasms + group. One hundred nine electrodes are implanted in total, consisting of 93 subdural grids over the left frontoparietotemporal region, two 4-contact strip electrodes placed over the left frontal pole (strip A) and the left superior frontal gyrus (strip B), and two 4-contact depth electrodes (depth C and D) placed in the magnetic resonance imaging lesion. The yellow dashed lines indicate the resection margin (frontal lobectomy and temporoparietal corticectomy). Seventyfour of 109 electrodes were resected. The red closed circles represent the 46 electrodes with high-rate ripples (A), and the 32 electrodes with high-rate FRs (B). The white open circles virtually show the electrodes concealed by the dura on strip A, and the electrodes on depths C and D 
as described in a previous report. ${ }^{24}$ We subsequently calculated the number of electrodes with high-rate ripples/FRs (Figure 2).

Between the ES+ and ES- groups, we compared (1) the total number of electrodes, (2) the $\mathrm{OR}_{\text {(ripples/FRs) }}$ in all electrodes, and (3) the number of electrodes with high-rate ripples/FRs.

We also investigated the influence of age at surgery on $\mathrm{OR}_{\text {(ripples/FRs) }}$ in all electrodes.

Within the good seizure outcome group, we compared (1) the number of electrodes in the resected/unresected areas and (2) the $\mathrm{OR}_{\text {(ripples/FRs) }}$ in the resected/unresected areas between the ES+ and ES- groups.

\section{7 | MI}

MI was calculated in an automated fashion at each electrode using EEGLAB Toolbox PACTV.0.17 as described in a previous report. ${ }^{7,17}$

We measured the MI between 2 HFOs (ripples/FRs) and 5 different frequency bands of delta and theta activities (0.5-1 Hz, 1-2 Hz, 2-3 Hz, 3-4 Hz, 4-8 Hz). The MI was analyzed in (1) all electrodes in all 24 children and (2) the resected/unresected area in the 18 children with good seizure outcomes.

Subsequently we compared the $\mathrm{MI}_{\text {(ripples/FRs \& } 3-4 \mathrm{~Hz} \text { ) }}$ between the ES+ and ES- groups in (1) all electrodes in all 24 children and (2) the resected/unresected area in the 18 children with good seizure outcomes.

We also investigated the influence of age at surgery on $\mathrm{MI}_{\text {(ripples/FRs \& 3-4 Hz) }}$ in all electrodes.

We analyzed the correlation between $\mathrm{MI}_{\text {(ripples \& } 3-4 \mathrm{~Hz} \text { ) }}$ and $\mathrm{MI}_{(\mathrm{FRs}}$ \& 3-4 Hz) in the resected area for each case in the good seizure outcome group to demonstrate the difference of faster $\mathrm{HFO}$ coupling with 3 - to $4-\mathrm{Hz}$ oscillations between the ES+ and ES- groups.

\subsection{Statistical analysis}

All statistical analyses were performed with SPSS Statistics 22 (IBM, Armonk, NY, USA). We performed Mann-Whitney $U$ test between 2 groups and Steel-Dwass test for intergroup comparisons. Simple linear regression analysis was performed to evaluate the correlation between age at sur-

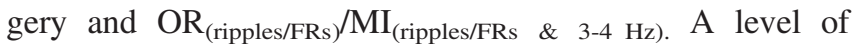
$P<.05$ was accepted as statistically significant.

\section{3 | RESULTS}

\section{1 | Clinical profiles}

Table 1 summarizes the clinical profiles of 24 children (10 females, 42\%). The ES+ group consisted of 10 (42\%) children. The ES - group consisted of $14(58 \%)$ children.

\section{Histogram of electrodes by occurrence rate (OR: / $\mathrm{min}$ ) of fast ripples (FRs) in ES+ and ES-}
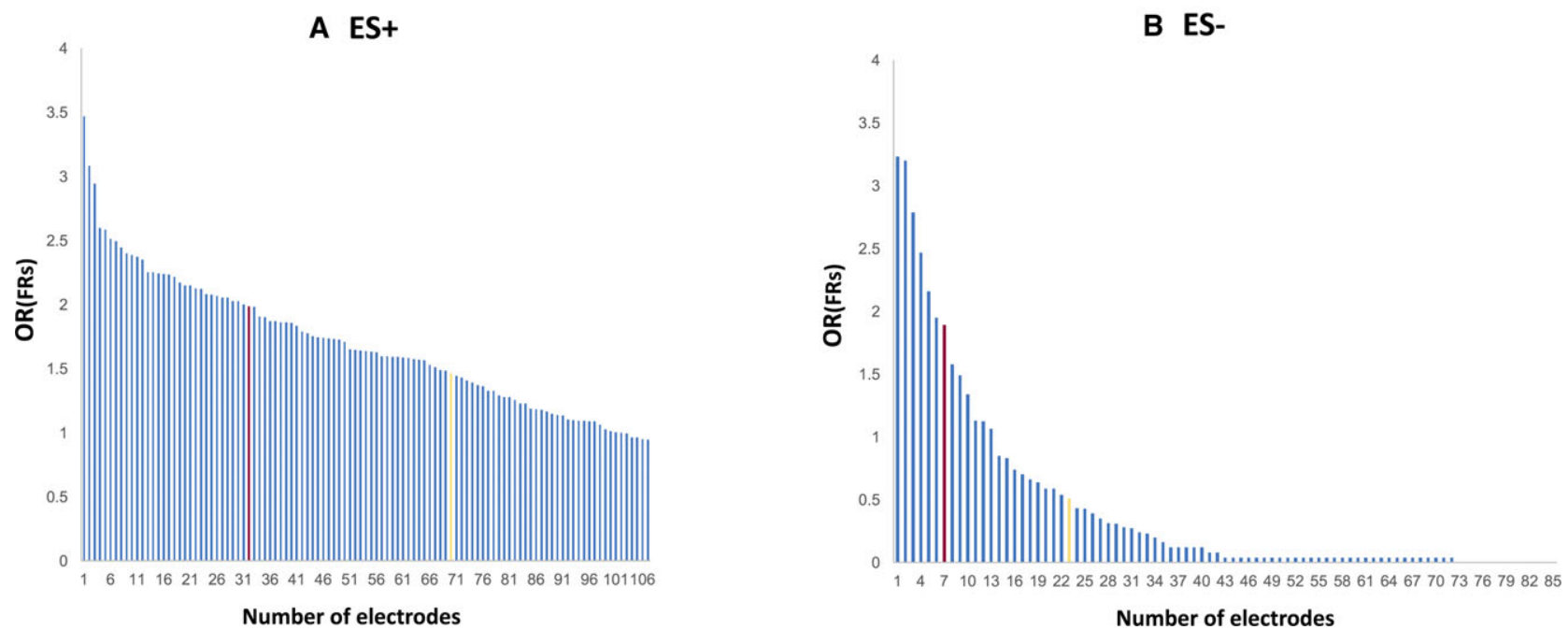

F IGURE 2 Histogram of electrodes by occurrence rate (OR; per minute) of fast ripples (FRs) in epileptic spasms (ES)+ and ES- subjects. The histograms show the distribution of electrodes ranked according to the OR) of FRs. A, Case \#7 in the ES+ group. B, Case \#13 in the ESgroup. The red and yellow lines divide the electrodes into 3 groups: high-rate FRs, medium-rate FRs, and low-rate FRs (defined by Ward's method). The high-rate FR group consists of 32 of 109 electrodes in Case \#7 of the ES+ group (A), and 7 of 92 electrodes in Case \#13 of the ES- group (B) 


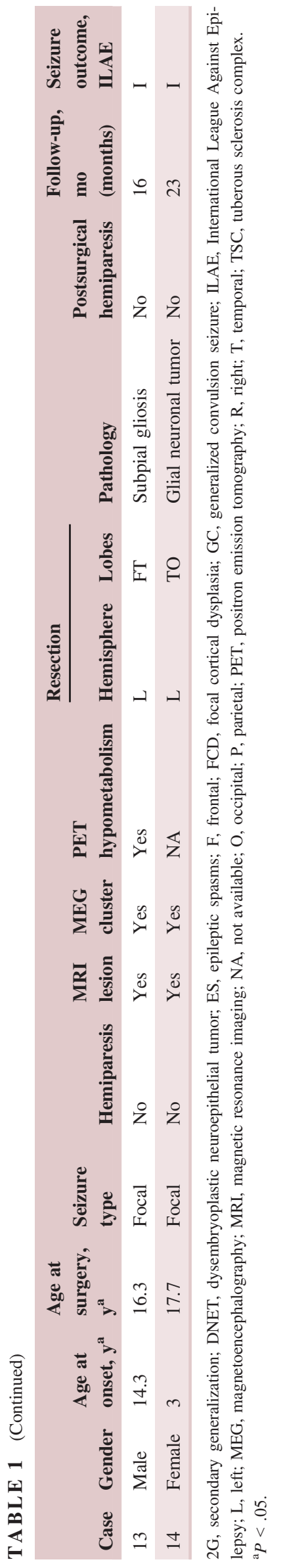

The age of seizure onset ranged from 0.2 to 6 years $($ mean $=2.7$ years) in the ES+ group, and from 0.3 to 14.3 years $($ mean $=5.4$ years $)$ in the ES- group. The age of seizure onset in the ES+ group was significantly younger than that in the ES- group $(P=.04)$.

The age at surgery ranged from 2.8 to 17 years (mean $=7.6$ years) in the ES + group, and from 1.8 to 17.7 years $($ mean $=12.2$ years) in the ES - group. The age at surgery in the ES+ group was significantly younger than that in the ES- group $(P=.01)$.

Two children $(20 \%, \# 2, \# 9)$ in the ES+ group and 2 children (14\%, \#6, \#7) in the ES- group had a history of infantile spasms. There were $6(60 \%)$ children in the ES+ group and 13 (93\%) children in the ESgroup with lesions detected on MRI. MEG showed clusters of MEG spike dipoles in $9(90 \%)$ children in the ES+ group and 13 (93\%) children in the ESgroup.

\section{2 | Resective surgery and pathology}

Five (50\%) children in the ES+ group and $8(57 \%)$ children in the ES - group underwent resective surgery in the left hemisphere.

Nine $(90 \%)$ children in the ES+ group and 5 (36\%) children in the ES - group underwent subtotal hemispherectomy consisting of frontotemporoparietal resection skipping the motor area.

In the ES+ group, histopathological examinations demonstrated focal cortical dysplasia (FCD) type I in 4 (40\%) children, III in $1(10 \%)$, astrogliosis in $4(40 \%)$, and oligodendrogliosis in $1(10 \%)$. There was no FCD type II in the ES+ group.

In the ES- group, histopathological examinations demonstrated FCD type I in 1 (7\%) child, II in $3(21 \%)$, III in 1 (7\%), astrogliosis in 1 (7\%), oligodendrogliosis in $3(21 \%)$, tuberous sclerosis complex in $3(21 \%)$, and glioneuronal tumor and normal pathology in $1(7 \%)$.

\section{3 | Seizure outcome}

The follow-up periods ranged from 14 to 56 months (mean $=33$ months). ILAE seizure outcome class Ia was achieved in $18(75 \%)$ children consisting of $7(70 \%)$ in the ES+ group and $11(79 \%)$ in the ES- group.

\subsection{Number of electrodes compared between the good and poor seizure outcome groups}

There were no significant differences in the number of electrodes in all electrodes or resected areas between the good and poor seizure outcome groups in total (good, 18; 
poor, 6), in the ES+ group (good, 7; poor, 3), and in the ES- group (good, 11; poor, 3; Table S2).

\subsection{Comparison between the ES+ and ES- groups in all electrodes}

\subsection{1 | Number of electrodes}

The total number of electrodes ranged from 85 to 121 (mean $\pm \mathrm{SD}=106.3 \pm 12.6$ ) in the $\mathrm{ES}+$ group and from 72 to 120 (mean $\pm \mathrm{SD}=105.2 \pm 13.1$ ) in the $\mathrm{ES}-$ group (Table 2).

\subsection{2 $\mid \mathbf{O R}_{\text {(ripples/FRs) }}$}

The $\mathrm{OR}_{(\mathrm{FRs})}$ in all electrodes (0.6-31.2, mean $\pm \mathrm{SD}$ $=8.7 \pm 10.4)$ in the ES + group was significantly higher than in the ES - group (0.4-10.2, mean $\pm \mathrm{SD}=3.0 \pm 2.7$; $P=.04$; Table 2). The $\mathrm{OR}_{\text {(ripples) }}$ in all electrodes ranged from 0.2 to 43.2 (mean $\pm \mathrm{SD}=12.0 \pm 12.9$ ) in the $\mathrm{ES}+$ group and from 0.9 to 12.7 (mean $\pm \mathrm{SD}=5.2 \pm 3.8)$ in the $\mathrm{ES}$ - group.

\subsection{3 | Number of electrodes with high-rate HFOs}

The number of electrodes with high-rate FRs (4-39, mean $\pm \mathrm{SD}=21.2 \pm 12.8)$ in the ES+ group was significantly higher than in the ES- group (2-40, mean $\pm \mathrm{SD}=10.0 \pm 10.5 ; P=.01$; Table 2$)$. The number of electrodes with high-rate ripples ranged from 4 to 46 (mean $\pm \mathrm{SD}=21.3 \pm 12.6$ ) in the ES+ group and from 2 to 40 (mean $\pm \mathrm{SD}=16.2 \pm 11.3$ ) in the $\mathrm{ES}-$ group.

TAB LE 2 Comparison between ES+ and ES- in all 24 children

\begin{tabular}{|c|c|c|c|}
\hline & $\mathbf{E S}+, \mathbf{n}=\mathbf{1 0}$ & $\mathbf{E S}-, \mathbf{n}=14$ & $P$ \\
\hline Total number of electrodes & $106.3 \pm 12.6$ & $105.2 \pm 13.1$ & .42 \\
\hline \multicolumn{4}{|l|}{$\mathrm{OR} / \mathrm{mm}$ in all electrodes } \\
\hline Ripples & $12.0 \pm 12.9$ & $5.2 \pm 3.8$ & .06 \\
\hline FRs & $8.7 \pm 10.4$ & $3.0 \pm 2.7$ & $.04^{\mathrm{a}}$ \\
\hline \multicolumn{4}{|c|}{ Number of electrodes with high-rate HFOs } \\
\hline Ripples & $21.3 \pm 12.6$ & $16.2 \pm 11.3$ & .17 \\
\hline FRs & $21.2 \pm 12.8$ & $10.0 \pm 10.5$ & $.01^{\mathrm{a}}$ \\
\hline \multicolumn{4}{|l|}{ MI in all electrodes } \\
\hline Ripples \& 3-4 HZ & $7.8 \pm 8.6$ & $3.2 \pm 1.9$ & .06 \\
\hline FRs \& 3-4 HZ & $1.1 \pm 1.0$ & $0.4 \pm 0.3$ & $.03^{\mathrm{a}}$ \\
\hline
\end{tabular}

ES, epileptic spasms; FR, fast ripple; HFO, high-frequency oscillation; MI, modulation index; OR, occurrence rate.

${ }^{\mathrm{a}} P<.05$.

\subsection{4 | Comparison of the MI between 2 HFOs (ripples/FRs) and 5 different frequency bands of delta and theta activities, in all electrodes}

In both the $\mathrm{ES}+$ and $\mathrm{ES}-$ groups, $\mathrm{MI}_{\text {(ripples/FRs \& } 3-4 \mathrm{~Hz})}$ was the highest among the 5 frequency bands (Figure 3). In the ES+ group, $\mathrm{MI}_{(\text {ripples/FRs \& 3-4 Hz) }}$ was significantly higher than $\mathrm{MI}_{\text {(ripples/FRs \& } 0.5-1 \mathrm{~Hz})}(P=.04)$. In the $\mathrm{ES}-$ group, there was no significant difference of $\mathrm{MI}_{\text {(ripples/FRs) }}$ among the 5 frequency bands.

\subsection{5 | MI $_{(\text {ripples/FRs \& 3-4 Hz) }}$}

The $\mathrm{MI}_{(\mathrm{FRs}}$ \& 3-4 Hz) in all electrodes $(0.1-3.4$, mean $\pm \mathrm{SD}=$ $1.1 \pm 1.0)$ in the ES+ group was significantly higher than in the ES - group (0.1-1.1, mean $\pm \mathrm{SD}=0.4 \pm 0.3 ; P=.03$; Table 2). The $\mathrm{MI}_{\text {(ripples \& 3-4 Hz) }}$ in all electrodes ranged from 1.0 to 27.0 (mean $\pm \mathrm{SD}=7.8 \pm 8.6$ ) in the $\mathrm{ES}+$ group and from 0.7 to 7.3 (mean $\pm \mathrm{SD}=3.2 \pm 1.9$ ) in the $\mathrm{ES}$ - group.

\subsubsection{Correlation between age at surgery and $\mathrm{OR}_{(\text {ripples/FRs) }} / \mathrm{MI}_{(\text {ripples/FRs \& 3-4 Hz) }}$}

In the ES+ group, there was no significant correlation between age at surgery and $\mathrm{OR}_{\text {(ripples/FRs) }}(r=.25$, $P=.49 / r=.22, P=.54)$. In the $\mathrm{ES}-$ group, there was no significant correlation between age at surgery and $\mathrm{OR}_{\text {(ripples/FRs) }}(r=.19, P=.51 / r=.36, P=.21$; Figure $\mathrm{S} 2)$.

In the ES+ group, there was no significant correlation between age at surgery and $\mathrm{MI}_{\text {(ripples/FRs \& 3-4 Hz) }}(r=.29$, $P=.42 / r=.21, P=.56)$. In the $\mathrm{ES}-$ group, there was no significant correlation between age at surgery and $\mathrm{MI}_{\text {(ripples/FRs \& 3-4 Hz) }}(r=.30, P=.30 / r=.03, P=.91)$.

\section{6. | Children with good seizure outcomes: comparison between the ES+ and ES- groups in the resected/unresected areas}

\subsubsection{Number of electrodes in the resected/ unresected areas}

The number of electrodes in the resected area (44-94, mean $\pm \mathrm{SD}=68.4 \pm 15.6)$ in the ES + group was significantly higher than in the ES- group (26-61, mean $\pm \mathrm{SD}=$ $46.3 \pm 12.1 ; P<.01$; Table 3$)$.

The number of electrodes in the unresected area (16-61, mean $\pm \mathrm{SD}=35.4 \pm 14.0)$ in the $\mathrm{ES}+$ group was significantly lower than in the ES - group (14-87, mean $\pm \mathrm{SD}=$ $60.0 \pm 18.9 ; P<.01)$.

\subsection{2 $\mathrm{OR}_{\text {(ripples/FRs) }}$ in the resected/ unresected areas}

The $\mathrm{OR}_{(\mathrm{FRs})}$ in the resected area (0.6-37.9, mean $\pm \mathrm{SD}=$ $14.7 \pm 13.2)$ in the ES+ group was significantly higher 
F IGURE 3 Modulation index (MI) of 5 frequency bands in all electrodes in epileptic spasms $(\mathrm{ES})+$ and $\mathrm{ES}-$. $\mathrm{MI}_{(\mathrm{FRs}}$ \& 3-4 $\left.\mathrm{Hz}\right)$ was significantly higher in the $\mathrm{ES}+$ group than in the ES- group $(P=.03 ; \mathrm{B})$. In both the ES+ and ES- groups,

$\mathrm{MI}_{\text {(ripples/FRs \& 3-4 Hz) }}$ was highest. In the $\mathrm{ES}+$ group, $\mathrm{MI}_{\text {(ripples/FRs \& 3-4 Hz) }}$ was significantly higher than $\mathrm{MI}_{\text {(ripples/FRs \& } 0.5-1 \mathrm{~Hz})}$ $(P=.04)$. In the ES - group, there was no significant difference of $\mathrm{MI}_{\text {(ripples/FRs) }}$ among the 5 frequency bands
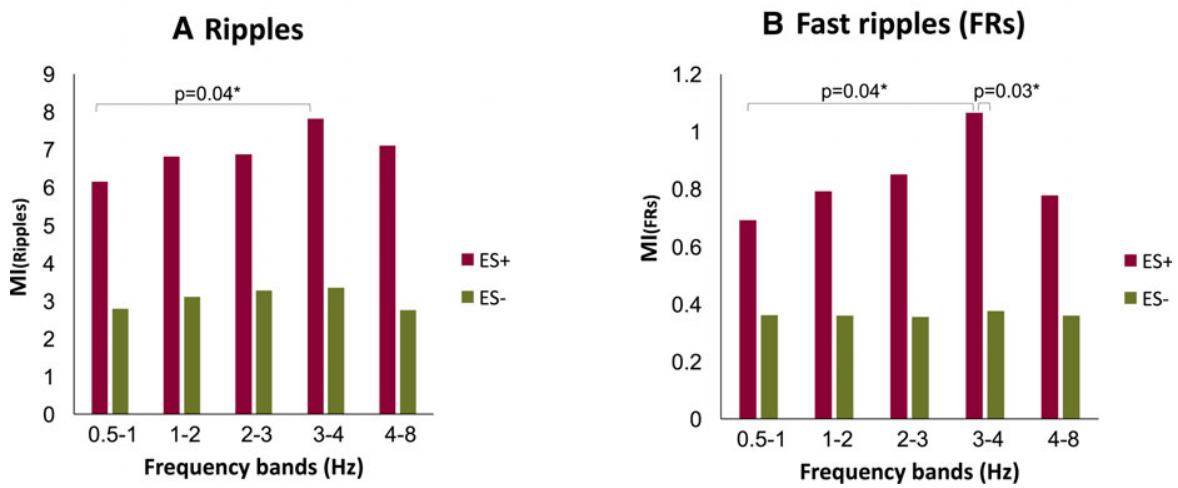

T A B LE 3 Comparison between ES+ and ES- in the good seizure outcome group

\begin{tabular}{|c|c|c|c|}
\hline & $\mathrm{ES}+, \mathbf{n}=7$ & $\mathbf{E S}-, \mathbf{n}=11$ & $P$ \\
\hline $\begin{array}{l}\text { Number of lobes including } \\
\text { the resected area }\end{array}$ & $3.1 \pm 0.4$ & $2.5 \pm 0.5$ & $<.01^{\mathrm{a}}$ \\
\hline $\begin{array}{l}\text { Number of electrodes in } \\
\text { the resected area }\end{array}$ & $68.4 \pm 15.6$ & $46.3 \pm 12.1$ & $<.01^{\mathrm{a}}$ \\
\hline $\begin{array}{l}\text { Number of electrodes in } \\
\text { the unresected area }\end{array}$ & $35.4 \pm 14.0$ & $60.0 \pm 18.9$ & $<.01^{\mathrm{a}}$ \\
\hline \multicolumn{4}{|l|}{$\mathrm{OR} / \mathrm{min}$ in the resected area } \\
\hline Ripples & $19.0 \pm 17.2$ & $10.0 \pm 8.6$ & .11 \\
\hline FRs & $14.7 \pm 13.2$ & $4.6 \pm 4.3$ & $.04^{\mathrm{a}}$ \\
\hline \multicolumn{4}{|c|}{$\mathrm{OR} / \mathrm{min}$ in the unresected area } \\
\hline Ripples & $10.8 \pm 11.3$ & $4.5 \pm 6.8$ & .11 \\
\hline FRs & $6.3 \pm 6.9$ & $2.8 \pm 4.4$ & .13 \\
\hline \multicolumn{4}{|l|}{ MI in the resected area } \\
\hline Ripples \& 3-4 Hz & $11.7 \pm 11.5$ & $5.2 \pm 4.4$ & .09 \\
\hline FRs \&3-4 Hz & $1.6 \pm 1.5$ & $0.5 \pm 0.3$ & $.04^{\mathrm{a}}$ \\
\hline \multicolumn{4}{|l|}{ MI in the unresected area } \\
\hline Ripples \& 3-4 Hz & $9.4 \pm 7.9$ & $3.0 \pm 3.2$ & .06 \\
\hline FRs \& 3-4 Hz & $1.3 \pm 0.9$ & $0.5 \pm 0.9$ & .05 \\
\hline
\end{tabular}

ES, epileptic spasms; FR, fast ripple; MI, modulation index; OR, occurrence rate. ${ }^{\mathrm{a}} P<.05$.

than in the ES- group (0.4-8.8, mean $\pm \mathrm{SD}=4.6 \pm 4.3$; $P=.04$; Table 3). The $\mathrm{OR}_{\text {(ripples) }}$ in the resected area ranged from 4.0 to 54.4 (mean $\pm \mathrm{SD}=19.0 \pm 17.2$ ) in the ES+ group and from 1.0 to 28.6 (mean $\pm \mathrm{SD}=$ $10.0 \pm 8.6)$ in the ES- group.

The $\mathrm{OR}_{(\mathrm{FRs})}$ in the unresected area ranged from 0.3 to 17.0 (mean $\pm \mathrm{SD}=6.3 \pm 6.9$ ) in the $\mathrm{ES}+$ group and from 0.4 to 15.8 (mean $\pm \mathrm{SD}=2.8 \pm 4.4$ ) in the $\mathrm{ES}$ - group. The $\mathrm{OR}_{\text {(ripples) }}$ in the unresected area ranged from 0.8 to 32.0 (mean $\pm \mathrm{SD}=10.8 \pm 11.3$ ) in the ES+ group and from 0.6 to 23.8 (mean $\pm \mathrm{SD}=4.5 \pm 6.8$ ) in the $\mathrm{ES}$ - group.

\subsection{3 | Comparison of the MI between 2 HFOs (ripples/FRs) and 5 different frequency bands in the good seizure outcome group}

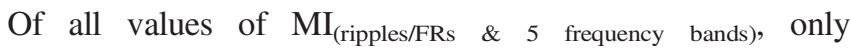
$\left.\mathrm{MI}_{(\mathrm{FRs}} \& 3-4 \mathrm{~Hz}\right)$ in the resected area was significantly higher in the $\mathrm{ES}+$ group than in the $\mathrm{ES}-$ group $(P=.04$; Figure S3).

In the resected area, $\mathrm{MI}_{\text {(ripples/FRs \& } 3-4 \mathrm{~Hz} \text { ) }}$ was the highest in both the ES+ and ES- groups.

In the unresected area, $\mathrm{MI}_{(\mathrm{FRs}}$ \& 3-4 $\left.\mathrm{Hz}\right)$ was the highest in both the $\mathrm{ES}+$ and $\mathrm{ES}-$ groups, $\mathrm{MI}_{\text {(ripples \& 3-4 Hz) }}$ was the highest in the ES+ group, and $\mathrm{MI}_{(\text {ripples \& } 1-2 \mathrm{~Hz} \text { ) }}$ was the highest in the ES- group. In both ES+ and ESgroups, there was no significant difference of $\mathrm{MI}_{\text {(ripples/FRs) }}$ among the 5 frequency bands.

\subsection{4 $\mid \mathrm{MI}_{(\text {ripples/FRs \& 3-4 Hz) }}$ in the resected/ unresected areas}

The $\mathrm{MI}_{(\mathrm{FRs}}$ \& 3-4 Hz) in the resected area $(0.1-4.5$, mean \pm $\mathrm{SD}=1.6 \pm 1.5$ ) in the ES+ group was significantly higher than in the ES- group (0.2-1.9, mean $\pm \mathrm{SD}=0.5 \pm 0.3$; $P=.04$; Table 3). The $\mathrm{MI}_{\text {(ripples \& 3-4 Hz) }}$ in the resected area ranged from 2.7 to 33.3 (mean $\pm \mathrm{SD}=11.7 \pm 11.5$ ) in the ES+ group and from 1.1 to 15.8 (mean $\pm \mathrm{SD}=$ $5.2 \pm 4.4)$ in the ES - group.

The $\left.\mathrm{MI}_{(\mathrm{FRs}} \& 3-4 \mathrm{~Hz}\right)$ in the unresected area ranged from 0.2 to 2.3 (mean $\pm \mathrm{SD}=1.3 \pm 0.9$ ) in the $\mathrm{ES}+$ group and from 0.1 to 3.3 (mean $\pm \mathrm{SD}=0.5 \pm 0.9$ ) in the $\mathrm{ES}$ - group. The $\mathrm{MI}_{\text {(ripples \& 3-4 Hz) }}$ in the unresected area ranged from 1.4 to 20.8 (mean $\pm \mathrm{SD}=9.4 \pm 7.9$ ) in the $\mathrm{ES}+$ group and from 0.4 to 11.7 (mean $\pm \mathrm{SD}=3.0 \pm 3.2$ ) in the $\mathrm{ES}-$ group.

\subsection{5 | Correlation of $\mathrm{MI}_{(\text {ripples \& 3-4 Hz) }}$ and $\mathrm{MI}_{(\mathrm{FRs} \& \text { 3-4 Hz) }}$ in the resected area}

In the ES+ group, there was a significant correlation between $\mathrm{MI}_{(\mathrm{ripples}}$ \& 3-4 Hz) and $\mathrm{MI}_{(\mathrm{FRs}}$ \& 3-4 Hz) $(r=.96)$ in 
each child $(P<.01$; Figure $\mathrm{S} 4)$. In the $\mathrm{ES}-$ group, there was a significant correlation between $\mathrm{MI}_{(\text {ripples } \& 3-4 \mathrm{~Hz})}$ and $\left.\mathrm{MI}_{(\mathrm{FRs}} \& 3-4 \mathrm{~Hz}\right)(r=.88)$ in each child $(P<.01)$. No overlapping of the $95 \%$ confidence interval between the ES+ and ES - groups was found. These findings revealed that FRs coupled more strongly with 3 - to $4-\mathrm{Hz}$ oscillations than ripples did in the ES+ group, in comparison to the $\mathrm{ES}-$ group.

\section{4 | DISCUSSION}

\subsection{Summary of findings}

In the $\mathrm{ES}+$ group $(\mathrm{n}=10)$, the $\mathrm{OR}_{(\mathrm{FRs})}$, the number of electrodes with high-rate FRs, and the $\mathrm{MI}_{(\mathrm{FRs}}$ \& 3-4 Hz) in all electrodes were significantly higher than those in the $\mathrm{ES}-$ group $(\mathrm{n}=14)$.

Within the good seizure outcome group $(n=18)$, the $\mathrm{OR}_{(\mathrm{FRs})}$ and the $\mathrm{MI}_{(\mathrm{FRs} \& 3-4 \mathrm{~Hz})}$ in the resected area in the ES+ group $(n=7)$ were significantly higher than those in the ES- group $(\mathrm{n}=11)$.

\subsection{Widespread epileptogenesis in ES}

The number of total electrodes with high-rate FRs in the ES+ group was significantly higher than that in the ESgroup.

Chugani et al. reported 50 of 65 patients with ES who underwent hemispherectomy, subtotal hemispherectomy, or multilobar resection based on positron emission tomography studies at Children's Hospital of Michigan between 1993 and 2014. ${ }^{6}$ Thirty-seven (74\%) of these patients achieved seizure freedom. In another study, 15 of 51 patients with ES secondary to FCD on MRI underwent multilobar resections. ${ }^{25}$ Twelve $(80 \%)$ of these patients became seizure-free. It has been demonstrated that a subset of children with drug-resistant ES who underwent more widespread resections are more likely to achieve seizure freedom than those who undergo limited resections, such as lesionectomy. ${ }^{5}$ Intracranial EEG in 11 children with drug-resistant ES confirmed that focal seizures associated with spasms might originate from multiple cortical $^{\text {areas. }^{26}}$ The analysis of interictal HFOs using IVEEG suggested that the seizure onset zone was larger in ES than in focal seizures. ${ }^{12}$ Resection of the areas demonstrating ictal HFOs, especially FRs, on IVEEG correlated with a good surgical outcome in children with ES. $^{27}$

In our cohort, electrodes with high-rate FRs in the ES+ group were widely distributed over multiple lobes in the unilateral hemisphere, indicating widespread epileptogenicity.

\section{3 | Severity of epileptogenesis in ES}

In the $\mathrm{ES}+$ group, $\mathrm{OR}_{(\mathrm{FRs})}$ and $\mathrm{MI}_{(\mathrm{FRs}}$ \& $\left.3-4 \mathrm{~Hz}\right)$ in the resected area were significantly higher than in the ESgroup. FRs were more prominent in both OR and MI than ripples in the ES+ group.

FRs have been proposed as a biomarker of the epileptogenic zone. ${ }^{9,28}$ The interictal high-rate FRs are a possible surrogate marker of the epileptogenic zone. ${ }^{12}$ Resection of brain regions containing HFOs, especially FRs, correlated with good seizure outcome using extraoperative electrocorticography in adult patients ${ }^{29}$ and intraoperative electrocorticography in pediatric patients. ${ }^{11}$ Our data suggested that $\mathrm{OR}_{(\mathrm{FRs})}$ in the ES+ group were significantly higher than those in the ES - group. High values of $\mathrm{OR}_{(\mathrm{FRs})}$ could be a marker of the severity of epileptogenesis in ES.

MI demonstrates the degree of coupling between the amplitude of HFOs and the phase of slow waves. ${ }^{30}$ Nonoda et al reported that epileptogenic HFOs may be more preferentially coupled with slow waves of 3-4 Hz. ${ }^{17}$ We reported that $\mathrm{OR}_{(\mathrm{HFOs})}$ and $\mathrm{MI}_{(\mathrm{HFOs}}$ \& $\left.3-4 \mathrm{~Hz}\right)$ could be valuable biomarkers to identify epileptogenic extramotor areas. ${ }^{7} \mathrm{We}$ analyzed the correlations between $\mathrm{MI}_{(\text {ripples \& } 3-4 \mathrm{~Hz})}$ and $\mathrm{MI}_{(\mathrm{FRs}}$ \& 3-4 Hz) at each electrode. In the ES+ group, FRs coupled more strongly with 3 - to $4-\mathrm{Hz}$ oscillations than ripples did, in comparison to the ES- group. The high values of $\mathrm{OR}_{(\mathrm{FRs})}$ and $\mathrm{MI}_{(\mathrm{FRs}}$ \& 3-4 Hz) represent severe epileptogenicity in drug-resistant ES.

\subsection{Mechanism of ES}

ES can be categorized as focal, generalized, or of combined onset in the ILAE seizure type classification. ${ }^{3}$ Based on our results and previous studies, ${ }^{1,4,6,15,26}$ we may speculate the 2 neurophysiological mechanisms of ES in which multilobar resections achieved seizure freedom: (1) ES originates from multifocal cortices, and (2) ES originates from combined cortex and subcortical regions.

1. The cortex: The cerebral cortex has been reported to be the generator of ES. ${ }^{4,6,15,26}$ Intracranial EEG showed that ES originate from multiple cortical areas. ${ }^{4,26}$ Scalp EEG demonstrated ictal fast oscillations indicative of the cortical origin of ES. ${ }^{14,31}$ Our data suggested that extensive resected area showing high values of $\mathrm{OR}_{(\mathrm{FRs})}$ and $\mathrm{MI}_{(\mathrm{FRs}}$ \& 3-4 Hz) achieved seizure freedom in children with drug-resistant ES.

2. The cortex and the subcortical region: Our study revealed significantly higher value of MI between FRs and slow oscillations in children with ES compared to children without ES. MI reflects the degree of stability of phase-amplitude coupling between HFOs and slow 
oscillations. ${ }^{30}$ Previous studies present slow oscillations as an important feature of ictal and interictal EEG in ES. ${ }^{1,14,24,32}$ We speculate that the slow oscillation component may associate with the difference between ES+ and ES-. In patients with ES who underwent corpus callosotomy, there were no significant changes in the bilateral distribution or any parameters of the slow waves. ${ }^{33}$ The slow waves in ES did not originate from the corticocortical pathway via the corpus callosum, but rather from the corticosubcorticocortical pathway. Vigevano et al proposed that cortical-subcortical interaction is essential for the generation of infantile spasms. ${ }^{1}$ High values of MI in our data may indicate that slow oscillations originating from the subcortical regions play an important role in the generation of ES. Sakuma et al reported that children with ES had a higher number of oligodendroglialike cells (OLCs) in the subcortical region including the gray-white matter junction and white matter than those without ES. ${ }^{34}$ They speculated that increased populations of OLCs in the subcortical region could actually be a subset of features responsible for the ES. We speculate that the interaction between the cortex and the subcortical regions provokes ES.

\section{5 | Limitations}

A small number of children were enrolled in our study, because we selected children who underwent multilobar resection. A subset of ES children underwent unilobar resection to control ES. ${ }^{8}$ The epileptogenic characteristics of HFOs between multilobar and unilobar resection for ES should be evaluated. We are currently identifying more ES+ and ES - children to differentiate the electrophysiological specifics of ES from those of focal seizures.

Because adults were not included in our study, we could not compare OR and MI between children and adults. Instead, we have analyzed the correlation of OR/MI and the age of the children (1.8-17.7 years) when they underwent surgery.

We categorized the electrodes into 3 clusters: highrate ripples/FRs, medium-rate ripples/FRs, and low-rate ripples/FRs, according to the $\mathrm{OR}_{\text {(ripples/FRs) }}$ by Ward's method, as it is a commonly used hierarchical clustering procedure. We simply demonstrated the distribution of electrodes according to the occurrence rate of HFOs. We do not indicate that the high-rate HFO cluster is epileptogenic in this study. The issue of thresholding to determine the epileptogenic HFOs is still under discussion. ${ }^{13}$ It would be of interest to compare various thresholding techniques to determine which has the highest validity.
Stereotactic EEG has the potential to delineate subcortical electrophysiological activity in ES in the future. Analysis of corticocortical evoked potential (CCEP) has demonstrated epileptic networks ${ }^{35,36}$ in patients with focal epilepsy. There are no reports investigating ES using CCEP. CCEP may reveal the widespread epileptogenicity of drug-resistant ES.

A recent study, utilizing preferred phase angles of coupling, delineates the epileptic network activities. ${ }^{37}$ Further studies considering preferred phase angles of coupling may explain the electrophysiological features and the detailed epileptic network activity of ES.

\section{ACKNOWLEDGMENTS}

Y.I. and H.O. were supported by EpLink, the epilepsy research program of the Ontario Brain Institute (OBI). The OBI is an independent nonprofit corporation, funded partially by the Ontario government. The opinions, results, and conclusions are those of the authors, and no endorsement by EpLink or the OBI is intended or should be inferred.

\section{DISCLOSURE}

None of the authors has any conflict of interest to disclose. We confirm that we have read the Journal's position on issues involved in ethical publication and affirm that this report is consistent with those guidelines.

\section{OR CID}

Yasushi Iimura (D) http://orcid.org/0000-0003-4263-5920

Eishi Asano iD http://orcid.org/0000-0001-8391-4067

\section{REFERENCES}

1. Vigevano F, Fusco L, Pachatz C. Neurophysiology of spasms. Brain Dev. 2001;23:467-72.

2. Wilmhurst JM, Gaillard WD, Vinayan KP, et al. Summary of recommendations for the management of infantile seizures: Task Force Report for the ILAE Commission of Pediatrics. Epilepsia. 2015;56:1185-97.

3. Fisher RS. The new classification of seizures by the International League against Epilepsy 2017. Curr Neurol Neurosci Rep. 2017;17:48.

4. Asano E, Juhász C, Shah A, et al. Origin and propagation of epileptic spasms delineated on electrocorticography. Epilepsia. 2005;46:1086-97.

5. Moseley BD, Nickels K, Wirrell EC. Surgical outcomes for intractable epilepsy in children with epileptic spasms. J Child Neurol. 2012;27:713-20.

6. Chugani HT, Ilyas M, Kumar A, et al. Surgical treatment for refractory epileptic spasms: the Detroit series. Epilepsia. 2015;56:1941-9. 
7. Iimura Y, Jones K, Hattori K, et al. Epileptogenic high-frequency oscillations skip the motor area in children with multilobar drugresistant epilepsy. Clin Neurophysiol. 2017;128:1197-205.

8. Barba C, Mai R, Grisotto L, et al. Unilobar surgery for symptomatic epileptic spasms. Ann Clin Transl Neurol. 2016;4:36-45.

9. Bragin A, Engel J Jr, Wilson CL, et al. Hippocampal and entorhinal cortex high-frequency oscillations $(100-500 \mathrm{~Hz})$ in human epileptic brain and in kainic acid-treated rats with chronic seizures. Epilepsia. 1999;40:127-37.

10. Jacobs J, Zijlmans M, Zelmann R, et al. High-frequency electroencephalographic oscillations correlate with outcome of epilepsy surgery. Ann Neurol. 2010;67:209-20.

11. Wu JY, Sankar R, Lerner JT, et al. Removing interictal fast ripples on electrocorticography linked with seizure freedom in children. Neurology. 2010;75:1686-94.

12. Akiyama T, McCoy B, Go CY, et al. Focal resection of fast ripples on extraoperative intracranial EEG improves seizure outcome in pediatric epilepsy. Epilepsia. 2011;52:1802-11.

13. Höller Y, Kutil R, Klaffenböck L, et al. High-frequency oscillations in epilepsy and surgical outcome. A meta-analysis. Front Hum Neurosci. 2015;9:574.

14. Kobayashi K, Oka M, Akiyama T, et al. Very fast rhythmic activity on scalp EEG associated with epileptic spasms. Epilepsia. 2004;45:488-96.

15. Kobayashi K, Akiyama T, Oka M, et al. A storm of fast (40$150 \mathrm{~Hz}$ ) oscillations during hypsarrhythmia in West syndrome. Ann Neurol. 2015;77:58-67.

16. Canolty RT, Edwards E, Dalal SS, et al. High gamma power is phase-locked to theta oscillations in human neocortex. Science. 2006;313:1626-8.

17. Nonoda Y, Miyakoshi M, Ojeda A, et al. Interictal high-frequency oscillations generated by seizure onset and eloquent areas may be differentially coupled with different slow waves. Clin Neurophysiol. 2016;127:2489-99.

18. Ochi A, Otsubo H, Donner EJ, et al. Dynamic changes of ictal high-frequency oscillations in neocortical epilepsy: using multiple band frequency analysis. Epilepsia. 2007;48:286-96.

19. Zelmann R, Zijlmans M, Jacobs J, et al. Improving the identification of high frequency oscillations. Clin Neurophysiol. 2009; 120:1457-64.

20. Staba RJ, Wilson CL, Bragin A, et al. High-frequency oscillations recorded in human medial temporal lobe during sleep. Ann Neurol. 2004;56:108-15.

21. Bagshaw AP, Jacobs J, LeVan P, et al. Effect of sleep stage on interictal high-frequency oscillations recorded from depth macroelectrodes in patients with focal epilepsy. Epilepsia. 2009;50:617-28.

22. Wieser HG, Blume WT, Fish D, et al. ILAE Commission Report. Proposal for a new classification of outcome with respect to epileptic seizures following epilepsy surgery. Epilepsia. 2001;42:282-6.

23. Otsubo H, Ochi A, Imai K, et al. High-frequency oscillations of ictal muscle activity and epileptogenic discharges on intracranial EEG in a temporal lobe epilepsy patient. Clin Neurophysiol. 2008;119:862-8.

24. Akiyama T, Akiyama M, Kobayashi K, et al. Spatial relationship between fast and slow components of ictal activities and interictal epileptiform discharges in epileptic spasms. Clin Neurophysiol. 2015;126:1684-91.

25. Kang JW, Rhie SK, Yu R, et al. Seizure outcome of infantile spasms with focal cortical dysplasia. Brain Dev. 2013;35:816-20.

26. de la Vaissière S, Milh M, Scavarda D, et al. Cortical involvement in focal epilepsies with epileptic spasms. Epilepsy Res. 2014;108:1572-80.

27. Nariai H, Nagasawa T, Juhász C, et al. Statistical mapping of ictal high-frequency oscillations in epileptic spasms. Epilepsia. 2011;52:63-74.

28. Staba RJ, Wilson CL, Bragin A, et al. Quantitative analysis of high-frequency oscillations $(80-500 \mathrm{~Hz})$ recorded in human epileptic hippocampus and entorhinal cortex. J Neurophysiol. 2002;88:1743-52.

29. Jacobs J, Levan P, Châtillon CE, et al. High frequency oscillations in intracranial EEGs mark epileptogenicity rather than lesion type. Brain. 2009;132:1022-37.

30. Canolty RT, Cadieu CF, Koepsell K, et al. Detecting eventrelated changes of multivariate phase coupling in dynamic brain networks. J Neurophysiol. 2012;107:2020-31.

31. Kobayashi K, Endoh F, Toda Y, et al. Occurrence of bilaterally independent epileptic spasms after a corpus callosotomy in West syndrome. Brain Dev. 2016;38:132-5.

32. Fusco L, Vigevano F. Ictal electroencephalographic findings of spasms in West syndrome. Epilepsia. 1993;34:671-8.

33. Honda R, Saito Y, Okumura A, et al. Characterization of ictal slow waves in epileptic spasms. Epileptic Disord. 2015;17:425-35.

34. Sakuma S, Halliday WC, Nomura R, et al. Increased subcortical oligodendroglia-like cells in pharmacoresistant focal epilepsy in children correlate with extensive epileptogenic zones. Epilepsia. 2016;57:2031-8.

35. Iwasaki M, Enatsu R, Matsumoto R, et al. Accentuated corticocortical evoked potentials in neocortical epilepsy in areas of ictal onset. Epileptic Disord. 2010;12:292-302.

36. Enatsu R, Gonzalez-Martinez J, Bulacio J, et al. Connectivity of the frontal and anterior insular network: a cortico-cortical evoked potential study. J Neurosurg. 2016;125:90-101.

37. Song I, Orosz I, Chervoneva I, et al. Bimodal coupling of ripples and slower oscillations during sleep in patients with focal epilepsy. Epilepsia. 2017;58:1972-84.

\section{SUPPORTING INFORMATION}

Additional Supporting Information may be found online in the supporting information tab for this article.

How to cite this article: Iimura Y, Jones K, Takada $\mathrm{L}$, et al. Strong coupling between slow oscillations and wide fast ripples in children with epileptic spasms: Investigation of modulation index and occurrence rate. Epilepsia. 2018;59:544-554. https://doi.org/10.1111/epi.13995 